\title{
Análisis y seguimiento de pacientes con tumores mediastinales egresados de los hospitales metropolitanos de Costa Rica durante 1996
}

\author{
Marcela Navarro Flores ${ }^{1}$, Luis García Gutiérrez², Carlos Salazar Vargas ${ }^{3}$
}

Resumen: En nuestro país se ha investigado poco sobre los tumores mediastinales, los clínicos tenemos la sensación de que estamos viendo cada vez más enfermos con estos tumores y que estos son mas frecuentemente malignos.

Objetivo: estudiar todos los pacientes nuevos egresados en todo el país con un tumor mediastinal, y continuar su seguimiento por 5 años. A raíz de ello sacar conclusiones epidemiológicas y de su comportamiento de acuerdo al tratamiento administrado.

Material y métodos: todos los pacientes egresados de los hospitales metropolitanos de adultos de Costa Rica durante 1996 con tumor mediastinal fueron estudiados y seguidos por 5 años completos.

Resultados: hubo 101 pacientes, 58 mujeres y 43 hombres, 52 tuvieron tumores malignos y 48 benignos. Las mujeres tuvieron más timomas, quistes y tumores neurogénicos,y los varones más de células germinales. La incidencia nacional para la población de 1996 fue de 2.9 y la de San José fue de 5.18 ajustada para su población, pero Limón, Puntarenas y Guanacaste tuvieron tasas menores que el promedio nacional. Todos los tumores benignos, los timomas y los neurogénicos malignos fueron resecados, aunque a los últimos se asoció radio y quimioterapia. Para algunos de células germinales se utilizó quimioterapia así como para los linfomas. Durante el período de seguimiento fallecieron 6 pacientes, todos con enfermedad maligna, y de los 46 vivos 5 han desarrollado metástasis.

Conclusión: la incidencia de tumores mediastinales en nuestro medio es alta comparada con la literatura internacional, asi como la incidencia de malignidad en los mismos. Estos datos son mas aparentes para la provincia de San José. Se muestran los hallazgos en relación con diferentes variables y la respuesta al tratamiento.

La radiografía convencional de tórax sigue siendo fundamental en el diagnóstico de estos tumores.

Descriptores: tumores, mediastino, linfoma, timoma, teratoma, coriocarcinoma, seminoma.

Recibido: 11 de marzo, 2003

Aceptado: 22 de abril, 2003

\footnotetext{
Abreviaturas: TM:tumor de mediastino; TCG:tumores de células germinales, TN: tumores neurogénicos, LH: linfoma de Hodgkin, TAC: tomografía axial computadorizada; US: ultrasonido; RM: resonancia magnética.

Médico General

Dirección de Compra de Servicios de Salud, Gerencia Administrativa, C.C.S.S.

Cirugía de Tórax y Cardiovascular, Hospital R. A. Calderón Guardia, C.C.S.S.

Correspondencia: Carlos Salazar V., apartado 1826 - 1250, Escazú, Costa Rica.
}

En el embrión no existe el mediastino, el cuello y el abdomen están comunicados por una área intermedia que luego se convertirá en el tórax. Alrededor de la $7^{\mathrm{a}}$. semana, aparece lo que se llama el mediastino primitivo después de la migración craneal o caudal de los órganos y de la formación de las cavidades celómicas (pericárdica y pleurales) y del diafragma, el que eventualmente separará ambas regiones ${ }^{1,2}$.

Durante la etapa embrionaria un grupo de blastómeros germinales que estaban en el polo cefálico migran hacia la zona lumbar, atravesando lo que será el mediastino, pudiendo quedar algunos rezagados de camino y eventualmente originar allí teratomas y a su vez, segmentos intestinales pueden originar duplicaciones intestinales o respiratorias, convirtiéndose posteriormente en masas quísticas del mismo ${ }^{3}$. 
Se convierte así esta región en una zona sumamente importarte y compleja, en la que dada la gran actividad y la presencia de diferentes órganos y tejidos, pueden originarse tumores de muy diversa estirpe, los que precisamente por su localización tan escondida, son de diagnóstico difícil.

El mediastino es un espacio limitado arriba por la entrada torácica, el diafragma por abajo, el esternón por delante, la columna vertebral por atrás y por la pleura parietal a los lados ${ }^{4}$.

Los tumores de mediastino pueden ser de origen neoplásico, congénito o inflamatorio ${ }^{5}$; pueden manifestarse por una variedad de síntomas, según sea su efecto sobre los órganos y estructuras adyacentes de acuerdo a su localización o pueden causar cuadros sistémicos mediante sustancias o factores inmunológicos propios del tumor.

El mediastino puede ser dividido anatómicamente de varias maneras, si se analiza desde el punto de vista del radiólogo o desde el punto de vista del clínico ${ }^{6}$.

La división topográfica usual es en 2 grandes regiones: superior e inferior, divididas por una línea imaginaria que va de la unión del manubrio con el cuerpo del esternón al borde superior de la 4ta.vértebra torácica y la región inferior se subdivide a la vez en anterior, medio y posterior ${ }^{4,7}$. Sin embargo y por razones clínicas, se ha simplificado esta división y muchos prefieren compartamentalizar el mediastino en ánterosuperior, medio y posterior ${ }^{8,9}$. Como el médico conoce la anatomía de la región, sabe que órganos ocupan cada compartimiento y puede sospechar con cierta aproximación el origen de un tumor dado.

Las características clínicas y epidemiológicas de los TM han sido descritas a través de los reportes de la literatura internacional ${ }^{5,10}$. La información escrita por personas interesadas en el tema basada en la experiencia nacional es escasa y poco reciente ${ }^{11-13}$. Otro aspecto importante de subrayar es que los reportes oficiales no recogen toda la información sobre este tipo de patología. El Ministerio de Salud reporta sólo 24 casos de "tumores del timo, corazón y mediastino" para 1996, y una incidencia de 0.9 para hombres y de 0.4 para mujeres por cada 100000 habitantes ${ }^{14}$.

El objetivo del presente trabajo es analizar las características epidemiológicas y clínicas de los pacientes diagnosticados con TM vistos en nuestra población durante un año completo y documentar, además, su respuesta a la terapia empleada, siguiendo su evolución durante 5 años.

\section{Materiales y métodos}

Se seleccionaron los expedientes clínicos de todos los pacientes egresados por primera vez con diagnóstico de TM, de los hospitales generales de referencia del área metropolitana (Hospital México, Hospital R.A Calderón Guardia y Hospital San Juan de Dios), debido a que sus áreas de atracción cubren todo el territorio nacional y además porque estas enfermedades son tratadas solamente en estos centros, por lo que estudiando los egresos de dichos nosocomios se estudia lo que acontece en todo el país. Los pacientes fueron atendidos desde el $1^{\circ}$ de enero al 31 de diciembre de 1996 y su evolución fue seguida hasta completar cinco años del diagnóstico.

Se elaboró un documento basado en el "Instructivo para propuestas de Investigación” del Hospital México exponiendo el objetivo principal y los secundarios de este estudio y se presentó ante los Comités de Ética e Investigación de cada uno de los hospitales generales del área metropolitana ${ }^{15}$. Cada comité otorgó el permiso correspondiente para proseguir con la investigación.

Inicialmente se pensó realizar un trabajo comprehensivo que incluyera niños y adultos y para tal fin se consultó al Departamento de Bioestadística del Hospital Nacional de Niños Dr. Carlos Sáenz Herrera, pero no se reportó ningún niño diagnosticado con este tipo de tumores para el año de 1996.

Los códigos correspondientes a los TM se tomaron de la "Clasificación Internacional de Enfermedades" (CIE-10) de la Organización Mundial de la Salud ${ }^{16} \mathrm{y}$ fueron agrupados de acuerdo a la clasificación histológica descrita por Duane y Sabiston ${ }^{4}$.

La división mediastinal utilizada fue la de Neville y Hoffman, es decir, en tres compartimentos: antero-superior, medio y posterior ${ }^{8,9}$.

Se elaboró un cuestionario estándar para la recolección de la información de los expedientes seleccionados. Las variables recolectadas fueron: la edad del paciente al momento del diagnóstico, género, provincia de nacimiento y de procedencia, hábitos (tabaquismo o ingesta de bebidas etílicas), actividad laboral, sintomatología asociada, tipo histológico del tumor, método diagnóstico utilizado y tratamiento recibido, así como la última fecha de consulta.. La información fue tabulada y organizada para su estudio.

Para hacer el análisis se utilizaron las funciones estadísticas del Programa Excel de Windows 2000®, la significancia estadística se definió cuando la p<0,05.

\section{Resultados}

Durante el año de 1996, un total de 101 nuevos enfermos fueron egresados de los hospitales generales de referencia con diagnóstico de TM. Treinta y nueve pacientes $(38,6 \%)$ fueron tratados en el Hospital México, 37 (36,6\%) egresaron del Hospital R.A. Calderón Guardia y 24 (23,8\%) del Hospital San Juan de Dios.

Hubo 52 enfermos con tumores malignos y 49 con tumores benignos; utilizando como rango esperado la distribución porcentual reportada por Davis y Sabiston se obtiene un valor de $\mathrm{p}=0,00006^{4}$.

Sesenta y ocho por ciento de los TM se localizaron en el mediastino ántero-superior, 20,8\% estaban en el compartimiento posterior y $9.9 \%$ en el mediastino medio (Figura 1). 


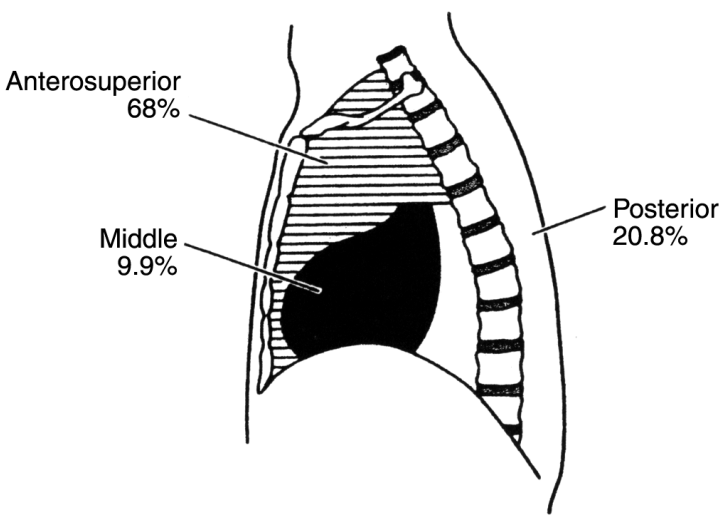

Figura No.1

Distribución anatómica de los Tumores de Mediastino

Un paciente que inicialmente se sospechó poseedor de TM finalmente fue egresado con el diagnóstico de "bocio intratorácico".

Sexo: Cincuenta y ocho pacientes $(57.42 \%)$ eran mujeres y 43 pacientes $(42.57 \%)$ eran hombres $(\mathrm{p}=0.196)$. Treinta y ocho mujeres presentaron tumores benignos y 20 presentaron tumores malignos $(\mathrm{p}=0,691)$. Por otro lado, 12 hombres se presentaron con TM benignos y 31 con malignos $(p=0,539)$.

En el cuadro \# 1 se puede ver la distribución por sexos en relación con la histología. Nótese como las mujeres tuvieron más timomas ( 15 vs. 4 ) y TN (13 vs. 2 ), en tanto que los TCG, los de origen mesenquimatoso, los linfomas y los quistes tuvieron una incidencia igual por sexos.

Edad: En esta serie se encontraron 20 pacientes menores de 30 años y 81 pacientes por encima de esta edad. El promedio de edad de las mujeres fue de 47 años 9 meses y el de los hombres de 47 años 2 meses.

El promedio de edad para las mujeres con TM benignos fue de 44 años 8 meses y el promedio para aquellas con TM malignos fue de 53 años 7 meses.

El promedio de edad para los hombres con TM benignos fue de 47 años y el promedio para aquellos con TM malignos fue de 47 años 3 meses. En el cuadro \#2 se puede ver la distribución de los TM en relación con los grupos etáreos.

Al separar los casos por décadas de edad, los grupos se vuelven pequeños, pero se hace evidente que los TN son bastantes constantes aunque de baja incidencia. Los linfomas son también constantes durante las primeras 5 décadas pero exhiben un pico en la 7 ta.

Los TCG malignos fueron más frecuentes de la $3^{\mathrm{a}}$ a la $5^{\mathrm{a}}$ década.

Sitio de nacimiento y procedencia: Treinta y cinco pacientes $(34,7 \%)$ habían nacido en San José, 20 (19,8\%) en Alajue-

\begin{tabular}{|lccc|}
\hline \multicolumn{4}{|c|}{$\begin{array}{c}\text { Cuadro 1 } \\
\text { Distribución porcentual de los TM según tipo } \\
\text { histológico y sexo }\end{array}$} \\
\hline Tipo Histológico & Total & Hombres & Mujeres \\
\hline Tumor de Células Germinales & $29 \%$ & $18 \%$ & $11 \%$ \\
Linfomas Primarios & $25 \%$ & $12 \%$ & $13 \%$ \\
Timomas & $19 \%$ & $4 \%$ & $15 \%$ \\
Quistes & $5 \%$ & $2 \%$ & $3 \%$ \\
Tumor Neurogénico & $15 \%$ & $2 \%$ & $13 \%$ \\
Tumor Mesenquimatoso & $7 \%$ & $4 \%$ & $3 \%$ \\
\hline Total & $100,00 \%$ & $42,00 \%$ & $58,00 \%$ \\
*Para analizar estrictamente tumores mediastinales se eliminó un \\
paciente con bocio intratorácico \\
\hline
\end{tabular}

\begin{tabular}{|lcccccccc|}
\hline \multicolumn{7}{|c|}{$\begin{array}{c}\text { Cuadro 2 } \\
\text { Distribución de los TM según edad }\end{array}$} \\
\hline Tipo histológico & $<20$ & $21-30$ & $31-40$ & $41-50$ & $51-60$ & $>60$ & Total \\
\hline Tumor de Células & 6 & 4 & 8 & 6 & 1 & 4 & 29 \\
Germinales & 1 & 3 & 1 & 4 & 4 & 12 & 25 \\
Linfoma & 0 & 0 & 1 & 4 & 5 & 9 & 19 \\
Timoma & 0 & 3 & 4 & 3 & 4 & 1 & 15 \\
Tumor Neurogénico & 0 & 0 & 2 & 2 & 0 & 2 & 6 \\
Tumor mesenquimatoso & 0 & 3 & 0 & 1 & 1 & 1 & 6 \\
Quistes & 7 & 13 & 16 & 20 & 15 & 29 & 100 \\
\hline Total & 7 & & & & & & \\
\hline
\end{tabular}

la y Cartago, $9(9,8 \%)$ en Heredia, $5(5 \%)$ en Puntarenas y Limón, 4 (4\%) en Guanacaste, y 3 (3\%) en Nicaragua.

La incidencia nacional para 1996 de TM de acuerdo con estos datos, para una población de 3489287 según reporta la Dirección Actuarial y Planificación Económica de la CCSS para este año, fue entonces de 2.9 por 100000 habitantes ${ }^{17}$.

Sesenta y cuatro pacientes (63\%) provenían de San José, 15 (15\%) de Alajuela, 10 (10\%) de Cartago, 4 (4\%) de Heredia, $3(3 \%)$ de Puntarenas y Limón y 2 pacientes $(2 \%)$ venían de Guanacaste.

La tasa de incidencia por 100000 habitantes por provincia puede verse en el cuadro 3. La provincia de San José obtuvo una tasa de incidencia casi el doble de la tasa nacional, mientras que las provincias de Limón, Puntarenas y Guanacaste exhibieron tasas claramente inferiores a la nacional.

Datos Clínicos: Las manifestaciones clínicas, en general, fueron vagas e inespecíficas. La mayoría de los pacientes presentaron al menos un síntoma. El síntoma que más se presentó, en la serie en general, fue la tos $(72,3 \%)$, seguida de pérdida de peso $(44,6 \%)$ y fiebre $(42,6 \%)$. Solo 9 pacientes $(8,9 \%)$ no presentaron, del todo, sintomatología alguna.

En los pacientes con TM malignos los síntomas que más se presentaron fueron de igual manera la tos $(69,2 \%)$, pérdida de peso $(59,6 \%)$ y fiebre $(53,8 \%)$. Por su parte, en los pacientes 


\begin{tabular}{|lcc|}
\hline \multicolumn{3}{|c|}{ Cuadro 3 } \\
Tasa de incidencia por $\mathbf{1 0 0} \mathbf{0 0 0}$ habitantes de $\mathbf{1 9 9 6}$ \\
\hline Provincia & No. Casos & Tasa \\
\hline San José & 64 & 5.18 \\
Cartago & 10 & 2.61 \\
Alajuela & 15 & 2.42 \\
Heredia & 4 & 1.28 \\
Limón & 3 & 0.96 \\
Puntarenas & 3 & 0.83 \\
Guanacaste & 2 & 0.76 \\
\hline Nacional & 101 & 2.9 \\
\hline
\end{tabular}

con tumores benignos los síntomas que más frecuentes fueron: la tos $(75,5 \%)$, disfagia $(32,7 \%)$ y disnea $(30,6 \%)$. Cuadros 4 y 5 .

Síndromes asociados: Un síndrome febril fue relativamente común, no solo se vió en algunos pacientes con linfoma, como sería lo clásico, sino en otros pacientes con variedades benignas o malignas de TM. La fiebre fue de características variables y obviamente pudo deberse a fenómenos propios del tumor como necrosis, licuefacción, superinfección, a una reacción sistémica al mismo, o a colapso pulmonar por compresión.

Siete pacientes con timoma presentaron Miastenia Gravis, otros 2 se presentaron con Síndrome de vena cava superior, 1 con Síndrome de Horner y 1 tuvo ginecomastia bilateral (paciente de 44 años con disgerminoma).

Ocupación: Esta información se recolectó en 87 pacientes con TM que no eran de origen congénito, para buscar alguna posible asociación. De ellos $61 \%$ se desempeñaban como misceláneos, $21.8 \%$ como técnicos y $17.2 \%$ eran profesionales. De los 51 enfermos con TM malignos y en los que se contaba con esa información, $60.8 \%$ eran misceláneos, $25.5 \%$ eran técnicos y el $13.7 \%$ eran profesionales. Así la distribución ocupacional para todo el grupo (malignos y benignos) fue igual que la de los malignos.

Método inicial de diagnóstico y de estadiaje: Todos los pacientes fueron sometidos a una tele-radiografía de tórax convencional y ésta fue la que inició el proceso de atención, detectando la lesión en la mayoría de los pacientes, mientras que en otros, fue necesario la realización de otros exámenes de gabinete.

La tomo grafía axial (TAC) fue utilizada en 13 pacientes, la broncoscopía en 8 , la mediastinoscopía en 6 , el US en 4 y la RM en 1 paciente.

Método de obtención de tejido para estudio histopatológico: En 24 pacientes (35\%) se efectuó biopsia por aspiración guiada por TAC, mediastinoscopía en 19 (28\%), en 15 (22\%) biopsia por aspiración guiada por US, toracotomía en $6(9 \%)$, y broncoscopía en 4 (6\%).
Grupo y Rh: En 86 enfermos con TM no congénitos se contó con este dato; $36(41.8 \%)$ tenían grupo $\mathrm{A}+, 32(37.2 \%)$ $\mathrm{O}+, 10(11.6 \%) \mathrm{B}+$, y $8(9.3 \%) \mathrm{AB}+$. Al separar los grupos según la histología, se observa que los pacientes con TCG y linfomas seguían la incidencia usual $(\mathrm{O}+>\mathrm{A}+)$ pero los TN y los timomas se presentaron el doble de veces en pacientes con grupo sanguíneo $\mathrm{A}+(8 / 4$ y $9 / 4)$ que en pacientes con grupo $\mathrm{O}+$.

Tratamiento: Los 49 enfermos con patología benigna fueron tratados únicamente con cirugía, y de los 52 pacientes con TM malignos, 32 recibieron quimio y radioterapia y los restantes 20 pacientes fueron tratados con cirugía, quimio y radioterapia.

El tratamiento quirúrgico (resección) se realizó por esternotomía o por toracotomía según fuese la presentación del tumor.

Mortalidad y seguimiento: Los pacientes con patología benigna, toleraron bien la resección quirúr gica y la mayoría siguieron control en la consulta externa de su área de atracción, después de un período inicial de seguimiento en los hospitales generales.

\begin{tabular}{|lc|}
\hline \multicolumn{2}{|c|}{$\begin{array}{c}\text { Cuadro 4 } \\
\text { TM Malignos } \\
\text { Síntomas presentes al momento del diagnóstico }\end{array}$} \\
\hline \multicolumn{1}{|c}{ Síntomas } & Porcentaje de pacientes \\
\hline Tos & $\mathrm{n}=52$ \\
Pérdida de peso & $69.2 \%$ \\
Fiebre & $59.6 \%$ \\
Disnea & $53.8 \%$ \\
Hiporexia & $38.5 \%$ \\
Dolor paraesternal & $25.0 \%$ \\
Sudoración nocturna & $25.0 \%$ \\
Adenopatías & $23.1 \%$ \\
Mialgias & $19.2 \%$ \\
Disfonía & $15.4 \%$ \\
\end{tabular}

\begin{tabular}{|lc|}
\hline \multicolumn{2}{|c|}{$\begin{array}{c}\text { Cuadro 5 } \\
\text { TM Benignos } \\
\text { Síntomas presentes al momento del diagnóstico }\end{array}$} \\
\hline \multicolumn{1}{|c|}{ Síntomas } & Porcentaje de pacientes \\
$\mathrm{n}=49$ \\
\hline Tos & $75.5 \%$ \\
Disfagia & $32.7 \%$ \\
Disnea & $30.6 \%$ \\
Fiebre & $28.6 \%$ \\
Pérdida de peso & $28.6 \%$ \\
Hiporexia & $24.5 \%$ \\
Dolor paraesternal & $20.4 \%$ \\
Asintomáticos & $18.4 \%$ \\
Disfonía & $16.3 \%$ \\
Sudoración nocturna & $12.2 \%$ \\
\hline
\end{tabular}




\begin{tabular}{|c|c|c|c|c|c|c|}
\hline \multicolumn{7}{|c|}{$\begin{array}{c}\text { Cuadro No. } 6 \\
\text { Pacientes con TM malignos fallecidos durante el periodo en estudio }\end{array}$} \\
\hline & Paciente 1 & Paciente 2 & Paciente 3 & Paciente 4 & Paciente 5 & Paciente 6 \\
\hline Sexo & femenina & masculino & masculino & femenina & masculino & femenina \\
\hline Edad & 53 & 32 & 46 & 47 & 81 & 90 \\
\hline Tipo de Tumor & teratocarcinoma & Linfoma Hodgkin & coriocarcinoma & $\begin{array}{c}\text { Schwanoma } \\
\text { maligno }\end{array}$ & Linfoma Hodgkin & Linfoma Hodgkin \\
\hline Tratamiento & quimioterapia & no & $\begin{array}{l}\text { quimio y } \\
\text { radioterapia }\end{array}$ & $\begin{array}{l}\text { quimio y } \\
\text { radioterapia }\end{array}$ & $\begin{array}{l}\text { quimio y } \\
\text { radioterapia }\end{array}$ & $\begin{array}{l}\text { quimio y } \\
\text { radioterapia }\end{array}$ \\
\hline Año de muerte & 1996 & 1997 & 1997 & 1998 & 1999 & 2001 \\
\hline
\end{tabular}

Los datos relacionados con los pacientes con TM malignos que fallecieron durante los cinco años de seguimiento del estudio,se muestran en el cuadro \#6. Una paciente con TCG falleció durante el mismo año del diagnóstico, 2 en el año siguiente, y 1 en cada año subsiguiente de seguimiento. De los 46 supervivientes a los 5 años del diagnóstico, 5 han desarrollado enfermedad metastásica y se encuentran muy limitados desde el punto de vista funcional.

\section{Discusión}

El mediastino es una cavidad situada en el medio del tórax, sin fascias ni planos anatómicos, en el que se alojan estrechamente una serie de órganos vitales, unos de estirpe vascular, otros respiratorios, digestivos, linfáticos y nerviosos. La variedad histológica de estos órganos y tejidos es extensa, sin embargo, debido a que la localización topográfica de ellos se conoce, cuando se desarrolla un tumor mediastinal su tipo histológico puede, si no predecirse con certeza, al menos ser incluido con lógica en el diagnóstico diferencial de un determinado paciente.

Otros datos ayudan al clínico a afinar aún más las posibilidades y a escoger la mejor manera de orientar la pesquisa diagnóstica y planear entonces el tratamiento más efectivo.

En Costa Rica se ha investigado poco sobre estos tumores ${ }^{11-13}$ y por otro lado, los registros oficiales sobre esta materia son genéricos e incompletos. En la publicación "Incidencia y Mortalidad del cáncer en Costa Rica 1994-1996" el Ministerio de Salud reporta bajo el código \#164 ("timo, corazón y mediastino") sólo 24 enfermos para 1996, sin embargo, de acuerdo con los datos aquí presentados en ese año hubo 101 nuevos casos ${ }^{14}$. Por esta razón la incidencia, que para efectos de la publicación del Ministerio de Salud es del 0.9 por 100000, es en realidad de 2,9.

Llama la atención la amplia variación en las tasas de incidencia por provincias, pero creemos que por ahora no podemos dar alguna explicación lógica a estos resultados.

La búsqueda de alguna relación de la ocurrencia de uno de estos tumores con la ocupación de los enfermos fue infructuosa y de existir, saldrá a la luz solo con estudios mucho más detallados y profundos.
Se indagó, asimismo, la presencia o no de alguna relación de los TM con el grupo sanguíneo de los pacientes. Como bien se sabe el cáncer gástrico es más frecuente en personas del grupo A y se ha visto mayor agresividad en ciertos tumores ginecológicos en pacientes de este mismo grupo. ${ }^{17,18}$ Aunque los datos aquí expuestos son muy pequeños, los timomas y los TN fueron el doble de frecuentes en pacientes con grupo $\mathrm{A}+$ que en pacientes $\mathrm{O}+$, grupo sanguíneo que es más común en nuestro país ${ }^{19}$. Estas relaciones merecen estudiarse en series más grandes tanto locales como internacionales.

Es el sentir de los clínicos dedicados al tratamiento de estos enfermos, que la patología mediastinal ha aumentado en nuestro país y que hoy vemos más tumores malignos que en el pasado.

A manera de comparación, en 1980 Salazar et al reportó 33 pacientes vistos en el Hospital México durante los 10 años precedentes, es decir, 3.3 pacientes por año ${ }^{11}$; mientras que en la presente serie se incluyen 39 enfermos tratados en ese nosocomio, pero sólo durante 1996. Se observa entonces un aumento de más de 10 veces en la incidencia de casos por año, habiendo crecido la población solamente 1.55 veces durante estos 16 años ${ }^{21}$.

La incidencia de TM malignos es muy variable y va del $25 \%$ en la mayoría de las series hasta el $49 \%$ en otras pocas, como ocurrió en este grupo, en otras palabras, nuestra incidencia de malignidad es alta, aunque se mantiene dentro del rango reportado en la literatura ${ }^{4,22}$.

Es interesante notar que en la publicación nacional arriba mencionada de $1980^{11}$, sólo un tercio de los TM de un Hospital fueron malignos, en tanto, en esta serie conjunta de 3 centros los tumores malignos suman ya casi la mitad.

Esa tendencia ya había sido detectada, el Walter Reed Medical Center demostró un aumento estadísticamente significativo de los tumores malignos al comparar 2 épocas diferentes; ellos documentaron un aumento sobre todo de los tumores del compartimiento anterior, señalando específicamente a los linfomas como causantes de esos hallazgos ${ }^{22}$.

Otro dato interesante es la frecuencia de malignidad de acuerdo con la edad, algunos autores refieren que son más 
frecuentes los TM malignos en edades más tempranas ${ }^{13,22}$ mientras que otros apuntan lo contrario ${ }^{4}$.

En esta serie se encontraron porcentajes diferentes a los encontrados en otras series en cuanto a la localización anatómica de los TM. Los tumores más frecuentes se localizaron en el compartimiento ántero-superior, seguidos por el medio y finalmente el compartimiento posterior. Davis y Sabiston reportaron incidencias de 54, 26 y $20 \%{ }^{4}$, nosotros encontramos incidencias de 68, 21 y $10 \%$ respectivamente.

La distribución histológica aquí encontrada difiere de la ya aludida de 1980, donde las lesiones no neoplásicas y tímicas fueron más frecuentes, asimismo, nuestros datos difieren de otras series internacionales; en esta serie se encontraron más linfomas y TCG y menos TN y lesiones quísticas que las reportadas por otros autores ${ }^{4,9,10}$.

Los métodos de obtención de biopsias de masas mediastinales han evolucionado desde 1959 cuando Carlens describió la mediastinocopía, procedimiento usado mayoritariamente en el estadiaje del carcinoma pulmonar, evitando así efectuar una toracotomía sólo para tomar material histológico ${ }^{24}$. Debido a sus limitaciones, Mc Neill y Chamberlain propusieron entonces la mediastinotomía anterior, complementándose así el abordaje del mediastino ántero-superior ${ }^{25}$. Chiba y otros empezaron lue go a realizar biopsias de las masas mediante aspiración de material dirigido por ultrasonido y por TAC ${ }^{26}$. A los métodos anteriores se ha agregado la toracoscopía, abordaje que se inició como maniobra diagnóstica y luego, con los avances en anestesias y tecnología, se utiliza también como procedimiento terapéutico ${ }^{26,27}$.

Finalmente, creemos que es necesario la realización de nuevos estudios que continúen actualizando y ampliando los conocimientos que actualmente disponemos sobre los TM en Costa Rica, asimismo, consideramos que el presente estudio debe servir de base para futuros estudios.

Es muy importante enfatizar que los TM no se diagnostican si el médico tratante no piensa en ellos. El estudio más sencillo y barato, aparte de la historia clínica y el examen físico, es la tele-radiografía de tórax, examen que no debiera negársele a ningún paciente

\section{Abstract}

Summary: Even though mediastinal tumors have been rarely studied in Costa Rica, our impression as clinicians is that we are seen more patients with this type of growths and that these are more frequently malignant.

Objective: To study all the patients in the whole country discharged with the diagnosis of "mediastinal tumor" and to follow them for 5 full years. According to the findings epidemiological conclusions were drawn as well as the natural history of the tumors in relationship to the treatment prescribed.
Methods: All the patients discharged during 1996, from the 4 main referral centers from Costa Rica with a mediastinal tumor were studied and followed for 5 complete years.

Results: A total of 101 individuals were diagnosed, 58 women and 43 men, 52 had malignant tumors and 48 had benign tumors. Women had more thymomas, cysts, and neurogenic tumors and men had more germinal cell growths. The national incidence for the 1996 population was 2.9 and that for San Jose was 5.18 adjusted for its population then. However Limon, Puntarenas and Guanacaste had lower rates than the national average. All benign tumors, thymomas and malignant neurogenic tumors were resected, although the latter were also treated with chemo and radiotherapy if needed. For some of the germinal cell tumors and for lymphomas chemo and radiotherapy was prescribed as well. During the follow up period 6 patients died, all had malignant disease and as for remaining 46 that are still alive 5 had developed metastatic spread.

Conclusion: our incidente of mediastinal tumors and their malignancy rate is high in comparison with international reports found in the literature. These figures are more apparent for the province of San Jose. The findings are shown according to different variables and the tumor's response to the treatments administered.

The conventional chest $\mathrm{x}$-ray is still of paramount importance in the diagnosis of mediastinal tumors.

\section{Referencias}

1. Moore K.L. Desarrollo de cavidades corporales, mesenterios primitivos y diafragma. En: Moore K.L. (Ed.) Embriología clínica. México. McGraw-Hill Interamericana,1989; 174 - 186.

2. Esposito C.,Romeo C. Surgical anatomy of the mediastinum. Seminars in Pediatric Surgery, 1999; 8:50-53.

3. Ronson RS., Duarte I., Miller JI. Embriology and surgical anatomy of the mediastinum with clinical implications. Surg Clin NA 2000; 80:157-169.

4. Duane DR., Sabiston DC. The mediastinum. En: Sabiston DC. (Ed.) Textbook of Surgery. Philadelphia. W. B. Saunders. 1997. p. 19061929.

5. Strollo DC., Rosado ML, Jett JR. Primary mediastinal tumors. Chest 1997; 112: 511-522.

6. Park D.,Pierson D. General principles and diagnostic approach. Disorders of the mediastinum. En:Murray and Nadel (eds). Textbook of respiratory medicine. Philadelphia W.B. Saunders,2000; 2079 - 2085.

7. Leigh TF., Weens HS. The mediastinum. Illinois. Charles C. Thomas, 1959.

8. Neville WE. Topography of the mediastinum. En:Nyhaus LM., Baker RJ. Eds. Mastery of Surgery. Boston. Little Brown and Co.,1992; 363 -373 .

9. Hoffman OA., Gillespie DJ., Aughenbaugh GL., Brown LR. Primary mediastinal neoplasms. Mayo Clin Proc 1993; 68:880-891.

10. Cohen AJ., Thompson L., Edwards FH., Bellamy RF. Primary cysts and tumors of the mediastinum. Ann Thorac Surg 1991,51:378-384.

11. Salazar C., Soto L., Pucci J., Brenes F. Tumores de mediastino. Acta Med Cost., 1980; 23: 253 - 265.

12. Salazar C, Soto L, Pucci J. Brenes F. Tumores primarios del timo. Acta Med Cost, 1980; 23(1):43-47. 
13. Mainieri J, Jaramillo J. Tumores de Mediastino. En: Jaramillo (Ed). El cáncer en Costa Rica. Editorial UCR, 1989; 319-328.

14. Bartels R., Vargas RM. Incidencia y mortalidad del cáncer en Costa Rica. 1994-1996. Registro Nacional de Tumores, Departamento Centro de Información,Dirección de Desarrollo de la Salud, Ministerio de Salud, 1996.

15. Instructivo para presentar propuestas de investigación. CCSS. Unidad de Investigación Científica. Hospital México. CCSS, 1997.

16. Clasificación estadística Internacional de las enfermedades y problemas relacionados con la Salud. 10 ${ }^{a}$ Revisión. Organización Panamericana de la Salud. Oficina Sanitaria Panamericana. Oficina de la Organización Mundial de la Salud. 1995.

17. Neugut AI., Hayek M., Howe G. Epidemiology of gastric cancer. Semin Oncol 1996; 23(3):281-291

18. Marinaccio M., Traversa A., Cariaggia E. Blood groups of the ABO system and survival rate in gynecologic tumours. Minerva Ginecol 1995; 47(3):69-76.

19. Brenes R. Incidencia de grupos sanguíneos y factor Rho en Costa Rica. Acta Med Cost 1978; 21:289-293.

20. Caja Costarricense de Seguro Social. Dirección Actuarial y Planificación Económica; 1996. San José, Costa Rica.
21. Centro Centroamericano de Población, Instituto Nacional de Estadística y Censos. Costa Rica: estimaciones y proyecciones de población 1970 - 2100 actualizadas al año 2000 y evaluación de Censo 2000 y otras fuentes de información: informe metodológico. San José, Costa Rica; Centro Centroamericano de Población, 2002.

22. Cohen AJ., Thompson FH., Bellamy RF. Primary cysts and tumors of the mediastinum. Ann Thorac Surg 1991; 51:378-384.

23. Temes R.,Allen N.,Chavez T., Crowell R., Key C., Wernley J. Primary mediastinal malignancies in children: report of 22 patients and comparison to 197 adults. The Oncologist 2000; 5:179-184.

24. Carlens E. Mediastinoscopy: a method for inspection and tissue biopsy in the superior mediastinum. Chest 1959; 36: $343-352$.

25. Mc Neill TM., Chamberlain JM. Diagnostic anterior mediastinotomy. Ann Thorac Surg 1996; 2:532 - 539.

26. Inaco LM,Milanes JR, Fernandez A.,Samano A., Fernández PP., Filomeno LT., Jatene FB. Diagnosis and treatment of mediastinal tumors by thoracoscopy. Chest 2000; 117: $1787-1792$.

27. Alvarado EM., Hoffman C., Salazar C. Video-toracoscopía. Rev Med CR y CA; 1999 56(549): 155-160.

\section{AMC, Abril-Junio 2003, vol 45 (2)}

